\title{
FULL LINEARIZATION OF INDUCTION MOTORS VIA NONLINEAR STATE-FEEDBACK
}

\author{
Alessandro De Luca, Giovanni Ulivi \\ Dipartimento di Informatica e Sistemistica, Università di Roma "La Sapienza" \\ Via Eudossiana 18, 00184 Roma, Italy
}

Abstract

A novel approach to the control of induction motors is presented, based on differential-geometric concepts for the control of nonlinear systems. Structural properties of the model are pointed out and a proper selection of system outputs is indicated which yields, via static state-feedback, exact state linearization and input-output decoupling of the closed-loop system. The proposed approach has been used to design a controller for motor torque and flux. Simulation tests are included.

\section{Introduction}

The control of drives using an induction motor as actuator is a long standing and thoroughly irvestigated problem $[1,2,3]$. The nonlinear dynamic behaviour of the machine, which is a major aspect, and the demand for a high performance/cost ratio require the application of advanced control techniques.

The dynamic model of the induction motor is typically a bilinear one [3]. The natural variable to be controlled is the output torque which is a nonlinear function of the motor state variables.

Moreover, the control system has to be designed in order to keep limited, even during fast transients, both the machine flux and the current sinked from the inverter. In fact, when the modulus of the machine flux exceeds some threshold value, which depends on the machine characteristics, the motor operates in an improper way; besides, the usual mathematical model of the machine does not hold anymore. On the other hand, an inverter cannot source a current value which is higher than its rated one, even for short time intervals; therefore, limiting current transients in the motor has also a direct influence on the size of the inverter.

Among the many solutions proposed for this problem, most of them are based on schemes which control separately the motor flux and the produced torque. In some of them, known as "field oriented" or "vector" control [1,4], it is possible to obtain, under certain hypotheses, an approximately linear and decoupled relation between input and output variables; to this aim a proper selection of the state variables is used.

Other approaches have been described which take explicitly into account the nonlinear nature of the model, based on the application of optimal $[5,6]$ or adaptive $[7,8]$ control methodologies.

Recently, the nonlinear control theory based on the differentialgeometric approach has achieved valuable results in the area of feedback linearization and decoupling of the class of smooth nonlinear systems which are linear in the inputs $[9,10,11]$. The resulting control techniques are quite mature and have already found useful application in different areas like robotics [12] and flight control [13]. Interesting results have appeared also in the field of motor control [14].

In this paper, the use of differential-geometric concepts and control techniques for nonlinear systems is proven to be effective for the control of induction machines. Embedding the problem in a general framework, allows a systematic investigation of the structural properties of the system model that leads to an interesting solution. The paper is organized as follows. The nonlinear dynamic model of the motor is described in Section 2 . To make the presentation selfcontained, the main results that will be used on feedback linearization and input-output decoupling are briefly recalled in Section 3, together with the mathematical tools needed. Section 4 investigates the properties of the dynamic model of the induction machine which are relevant from a control point of view. The synthesis of an exact linearizing and decoupling state-feedback controller is described in detail. Some simulation tests are reported and discussed in Section 5.

\section{Dynamic model of the induction machine}

The dynamic behaviour of a voltage fed induction motor can be described by a set of four nonlinear differential equations, considering for it the well known two-phase equivalent machine representation [3]. As usual, some simplifying hypotheses are made, i.e. iron losses and magnetic circuits' saturation are neslected and an isotropic structure is assumed.

Different choices of two-dimensional vector variables may be used, describing the motor dynamics in tems of rotor and/or stator fluxes and/or currents. The projections of these vectors on a reference frame which is fixed to or rotates with respect to the stator windings can be assumed as state variables.

A convenient choice for the state consists in using the projections of the stator current and stator flux vectors on a (d,q)-axes reference frame having its $d$-axis always aligned with the desired direction of the stator flux vector and, therefore, rotating at the same angular speed. The $i_{d s}$ and $i_{q s}$ components of the stator current can be obtained on the basis of direct measurement, while the $\varphi_{d s}$ and $\varphi_{q s}$ flux components can be reconstructed by means of an asymptotic observer of reduced order, as shown in $[15,16]$.

The projections $v_{d s}$ and $v_{q s}$ of the supply voltage and the slip frequency $\omega_{S}$, i.e. the difference between the desired angular speed of the flux and the mechanical speed $\omega$, are assumed as input variables. The speed $\omega$ can be considered as a slowly varying parameter, being the mechanical dynamics much slower than the electromagnetic one.

Therefore, setting

$$
x=\left[i_{d s}, i_{q s}, \varphi_{d s}, \varphi_{q s}\right]^{\top}, \quad u=\left[v_{d s}, v_{q s}, \omega_{s}\right]^{\top} \text {, }
$$

the dynamic equations describing the motor behaviour are bilinear and can be written as:

$$
\dot{x}=f(x)+g(x) u
$$

where

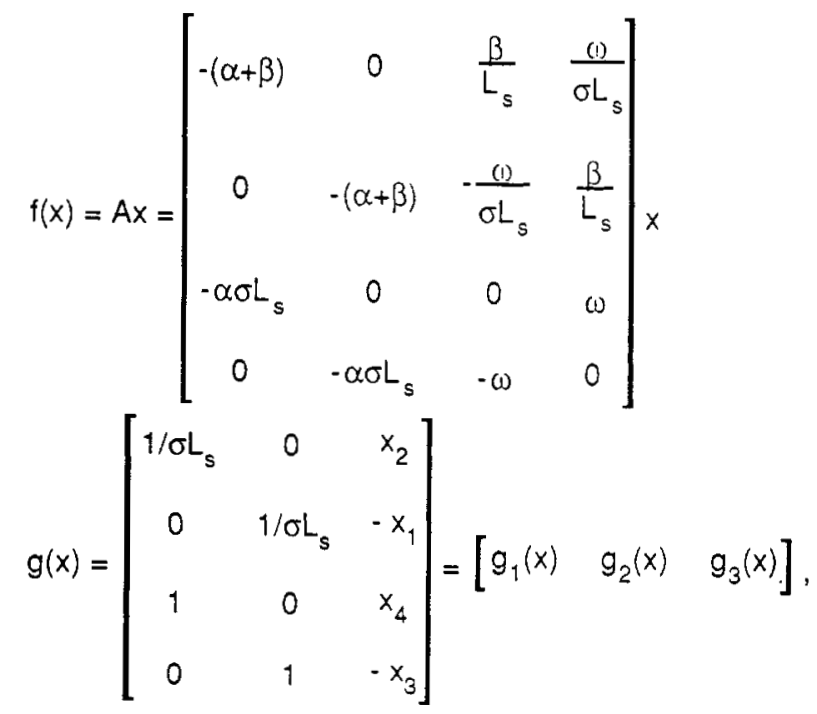


and $\alpha=R_{s} /\left(\sigma L_{s}\right), \beta=R_{r} /\left(\sigma L_{r}\right), \sigma=1-\left(M^{2} /\left(L_{s} L_{r}\right)\right)$. The parameters $R_{S}$ and $R_{r}$ are the stator and rotor resistances, $L_{S}$ and $L_{r}$ are the stator and rotor self-inductances and $M$ is the mutual inductance.

Quantities related to the rotor circuits can be expressed as linear combinations of the chosen state variables; in particular, the rotor flux components are given by:

$$
\varphi_{d r}=\left(\varphi_{d s}-\sigma L_{s} i_{d s}\right) L_{r} / M, \quad \varphi_{q r}=\left(\varphi_{q s}-\sigma L_{s} i_{q s}\right) L_{r} / M .
$$

The definition of the system outputs is connected with the control objectives. Usually in drives, the most important mechanical variable is the torque $T_{m}$ produced by the machine. Considering a motor with one pole pair, the torque is expressed as:

$$
T_{m}=\varphi_{d s} i_{q s}-\varphi_{q s} i_{d s} \text {. }
$$

To ensure correct motor operation, another controlled variable should be related to the motor flux and may be defined indifferently in terms of either stator or rotor fluxes; in fact, due to the tight coupling between the two windings, only a small difference arises between these vectors during normal operation.

Moreover, it is advisable to control also the q-component of the stator flux in order to guarantee the proper orientation of the reference frame with respect to which all motor variables are expressed. This generally allows some simplification in the implementation of the controller.

\section{Nonlinear control techniques}

Consider a multi-input multi-output nonlinear control system of the form:

$$
\begin{aligned}
& \dot{x}=f(x)+g(x) u=f(x)+\sum_{i=1}^{m} g_{i}(x) u_{i} \\
& y=h(x)=\left[\begin{array}{lll}
h_{1}(x) & \ldots & h_{m}(x)
\end{array}\right]^{\top}
\end{aligned}
$$

where the state $x \in \mathbb{R}^{n}$, the drift term $f(x)$ and each column $g_{i}(x)$ of the input matrix $g(x)$ are smooth vector fields, while each of the scalar outputs $h_{j}(x)$ is a smooth function. The system output definition is not unique; in any case, the attention is restricted to square systems i.e. with the same number of inputs and outputs.

In the last few years several control problems for nonlinear systems of the class (3.1-3.2) have been studied. The relevant feature of this class is that the control input appears linearly in the state equation; moreover, there is no instantaneous input-output relation. The main thrust has been in the direction of finding, if possible, transformations which map the nonlinear control problem into a linear one.

Consider the use of a static nonlinear state-feedback law of the form

$$
u=\alpha(x)+\beta(x) v
$$

with $\beta(x)$ nonsingular; the presence of $\beta(x)$ corresponds to a (statedependent) change of coordinates in the input space. The composition of (3.1-3.2) and (3.3) results in a closed-loop system which is still linear in the new input $v$ :

$$
\begin{aligned}
& \dot{x}=\tilde{f}(x)+\tilde{g}(x) \vee=[f(x)+g(x) \alpha(x)]+[g(x) \beta(x)] \vee, \\
& y=\tilde{h}(x)=h(x) .
\end{aligned}
$$

For systems without outputs, the feedback linearization problem consists of finding a feedback pair $(\alpha(x), \beta(x))$ such that there exists a state-space change of coordinates (a diffeomorphism)

$$
z=\Psi(x)=\left[\begin{array}{lll}
\Psi_{1}(x) & \ldots & \Psi_{n}(x)
\end{array}\right]^{\top}
$$

which transforms the state equation (3.4) of the closed-loop system into the Brunovski canonical form:

$$
\dot{z}=A z+B v \text {, }
$$

$A=$ block diag $\left[A_{1}, \ldots, A_{m}\right], B=$ block diag $\left[B_{1}, \ldots, B_{m}\right]$,

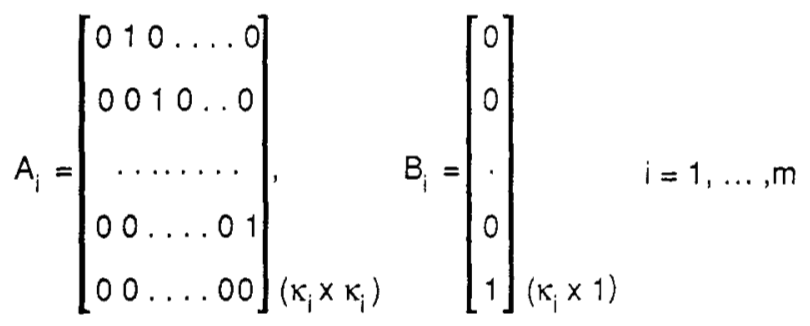

System (3.7) is a linear controllable one which is feedback equivalent to (3.1). The orders $\kappa_{i}$ of the blocks are the so-called controllability indices and stand for the lengths of the input-state integrator chains which are inirinsic to the system.

When the output equation (3.2) is explicitly considered, an appealing approach for reducing the complexity of the control problem is to achieve input-output decoupling i.e. finding a feedback pair $(\alpha(x), \beta(x))$ such that in the closed-loop system (3.4-3.5) the i-th output $y_{i}$ depends only on $v_{i}$, the $i$-th component of the new input $v$.

The two goals of exact linearization and input-output decoupling may indeed be pursued jointly. A full linearization problem can be stated where one looks for a feedback pair $(\alpha(x), \beta(x))$ and a coordinate transformation $z=\Psi(x)$ such that the closed-loop system (3.4-3.5) in the $z$ coordinates is expressed by (3.7) and

$$
y=C z \text {, }
$$

$$
\begin{aligned}
& C=\text { block diag }\left[C_{1}, \ldots, C_{m}\right], \\
& C_{i}=\left[\begin{array}{lllll}
1 & 0 & \ldots & 0 & 0
\end{array}\right]\left(1 \times k_{i}\right), \quad i=1, \ldots, m .
\end{aligned}
$$

The linear system (3.7-3.8) is a controllable and observable one. A complete introduction to the differential-geometric tools needed to state the main results for the previous problems can be found in [17]. Related to the state equations (3.1) a sequence of distributions $M_{k}$ may be defined in the following way:

$$
\begin{aligned}
& M_{0}(x)=\operatorname{sp}\left\{g_{1}(x), g_{2}(x), \ldots, g_{m}(x)\right\} \\
& M_{k}(x)=\operatorname{sp}\left\{M_{k-1}(x),\left[f, M_{k-1}\right](x)\right\}, \quad k=1,2, \ldots
\end{aligned}
$$

where $[f, M]$ denotes the Lie bracket between $f$ and each of the vector fields representing the distribution $M$. A necessary and sufficient condition for the solvability of the feedback linearization problem in a neighborhood $U \subset \mathbb{R}^{n}$ of a point $x_{0}$ is that for all $x \in U[9,11]$ :

(L1) $M_{k}(x)$ is an involutive distribution of constant rank, for $k=$ $0, \ldots, n-m ;$

(L2) $\operatorname{rank} M_{n-m}(x)=n$.

Note that this condition is stated locally; however, the property may hold everywhere in $\mathbb{R}^{n}$.

When the output equation (3.2) is considered, to each output $y_{i}$ is associated its characteristic number $\rho_{\mathrm{i}}$ defined as the largest integer such that for all $k<p_{i}$

$$
L_{g_{j}} L_{f}{ }^{k} h_{i}(x)=0, \quad \forall j \in\{1, \ldots, m\} .
$$

where $L_{\tau} h_{i}$ is the Lie derivative of the function $h_{j}$ w.r.t. a vector field $\tau$. The decoupling matrix $A(x)$ associated to the triple $(f, g, h)$ is defined as the mxm matrix with entries

$$
a_{i j}(x)=L_{g_{j}} L_{f} \rho_{i} h_{i}(x) \text {. }
$$

A necessary and sufficient condition for the solvability of the inputoutput decoupling problem in a neighborhood $U \subset \mathbb{R}^{n}$ of a point $x_{0}$ is that for all $x \in U[10]$ : 
(D1) $A(x)$ is nonsingular.

Again, this property may hold globally. When this result applies, a possible decoupling control is given by (3.3) with

$$
\alpha(x)=-A^{-1}(x) b(x) \quad \beta(x)=A^{-1}(x) \text {, }
$$

where $b(x)$ is an m-vector with entries

$$
b_{i}(x)=L_{i}^{\rho_{i+1}} h_{i}(x) \text {. }
$$

There is a nice feature of this decoupling law related to the linearity of the resulting closed-loop system. In fact, using the independent functions

$$
L_{f}^{k-1} h_{i}(x), \quad k=1, \ldots, p_{i}+1, \quad i=1, \ldots, m,
$$

as part of a new set of coordinates $z$, then the input-output behaviour of the closed-loop system $(3.1-3.2-3.10)$ is described by $m$ independent chains of integrators. Apart from this linear and decoupled part, the system may contain a sink, that is an unobservable and possibly nonlinear part. A condition which excludes automatically this situation is expressed algebraically as:

$$
\sum_{i=1}^{m}\left(p_{i}+1\right)=n
$$

The conditions (D1) and (D2) are only sufficient for full linearization, but very useful in practice. However, also necessiry and sufficient conditions exist for simultaneous state linearization and input-output decoupling [12].

\section{Nonlinear control of the induction motor}

\subsection{Feedback linearization}

In order to verify the feedback lirearizability of the induction motor state equations (2.1) no output needs to be defined for the system. Since $n=4$ and $m=3$, the general necessary and sufficient conditions (L1) and (L2) to be satisfied particularize in:

(L1) $M_{0}(x)=s p\left\{g_{1}(x), g_{2}(x), g_{3}(x)\right\}$ involutive and of constant rank;

(L2) $M_{1}(x)=\operatorname{sp}\left\{g_{i}(x),\left[f, g_{i}\right](x), i=1,2,3\right\}$ has dimension 4 .

From

$$
\left[g_{1}, g_{2}\right](x)=0,\left[g_{1}, g_{3}\right](x)=-g_{2}(x), \quad\left[g_{2}, g_{3}\right](x)=g_{1}(x)
$$

involutivity of $M_{0}$ follows. Moreover, it is easy to verify that

$$
\operatorname{dim} M_{0}(x)=3, \forall x \in V:=\left\{x \in \mathbb{R}^{4} \mid x_{1} \neq x_{3} / \sigma L_{s} \text { or } x_{2} \neq x_{4} / \sigma L_{s}\right\}
$$

so that (L1) holds in a neighborhood $U$ of any $x_{0}$ chosen in the given $V \subset \mathbb{R}^{4}$. Note that this subset of the state-space is contained in the one where the motor torque $T_{m}(x)=x_{2} x_{3}-x_{1} x_{4}$ is identically zero. Next, from

$\left[f, g_{1}\right](x)=\left[\begin{array}{c}{[\beta(\sigma-1)-\alpha] / \alpha_{s}} \\ -\omega / \sigma L_{s} \\ -\alpha \\ (j)\end{array}\right],\left[f, g_{2}\right](x)=\left[\begin{array}{c}\omega / \sigma L_{s} \\ {[\beta(\sigma-1)-\alpha] / \sigma L_{s}} \\ \omega \\ -\alpha\end{array}\right]$,

$\left[f, g_{3}\right](x)=0$,

it is possible to show that $\operatorname{dim} M_{1}(x)=4$ on $V$, so that aiso (L2) holds and therefore the system is feedback linearizable. To derive the structure of the feedback equivalent linear system, the controllability indices $\kappa_{i}, i=1,2,3$, associated to system (2.1) are needed. Since

$$
\begin{aligned}
& r_{0}:=\operatorname{dim} M_{0}(x)=3, \quad r_{1}:=\operatorname{dim} M_{1}(x)-\operatorname{dim} M_{0}(x)=1, \\
& r_{k}:=0, \quad \forall k \geq 2,
\end{aligned}
$$

and being the controllability indices computed as $\kappa_{i}:=\#\left\{r_{j} \mid r_{j} \geq i\right.$, $j=0,1, \ldots\}$ with $\kappa_{1} \geq \kappa_{2} \geq \kappa_{3}[9]$, it follows that

$$
\kappa_{1}=2, \quad \kappa_{2}=1, \quad \kappa_{3}=1
$$

Hence, there exists a static state-feedback $u=\alpha(x)+\beta(x) \vee$ and a state-space change of coordinates $z=\Psi(x)$, such that the closed-loop system assumes, in the $z$-coordinates, the following Brunovski controllable canonical form:

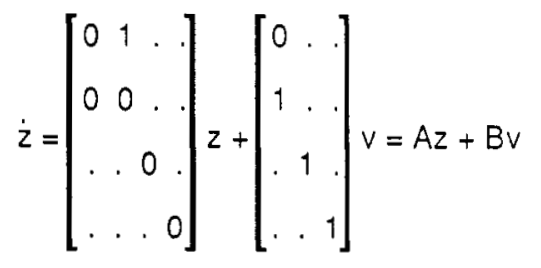

\subsection{Selection of system outputs}

The above canonical form results in the closed-loop system once the proper coordinates $z$ have been selected. In particular, in order to build the transformation $\Psi(x)$, three smooth functions $T_{i}(x), i=1,2,3$, have to be found in such a way that in $V[17]$ :

$$
L_{g_{j}} L_{f}^{k} T_{i}(x)=0, \quad j=1,2,3, \quad 0 \leq k \leq \kappa_{i}-2, \quad i=1,2,3 .
$$

The components $\Psi_{j}(x)$ of the transfomation are then recovered as:

$$
\begin{array}{ll}
\Psi_{1}(x)=T_{1}(x), & \Psi_{2}(x)=L_{1} T_{1}(x), \\
\Psi_{3}(x)=T_{2}(x), & \Psi_{4}(x)=T_{3}(x) .
\end{array}
$$

For $\mathrm{i}=1, \kappa_{1}=2$ and therefore it must be

$$
L_{g_{i}} T_{1}(x)=0, \quad j=1,2,3
$$

These conditions have to be verified for all $x \in V$. Since $\kappa_{2}=\kappa_{3}=1$, no conditions are imposed to $T_{2}(x)$ and $T_{3}(x)$. Moreover, the $T_{i}(x)$ 's can always be chosen in such a way that the matrix

$$
\left[\begin{array}{l}
L_{g} L_{f}^{k_{1}^{-1}} T_{1}(x) \\
L_{g} L_{f}^{k_{2}^{-1}} T_{2}(x) \\
L_{g} L_{f}^{x_{3}^{-1}} T_{3}(x)
\end{array}\right]
$$

is nonsingular in a neighborhood of a given $x_{0}$ chosen in $V$ [17]. Depending on the specific $T_{2}(x)$ and $T_{3}(x)$ used in (4.3), further singularities may be introduced in the control scheme. In fact, the nonsingularity of the above matrix may not hold globally on the whole $V$. Equations (4.2) develop into the following set of partial differential equations (PDE's):

$$
\begin{aligned}
& \frac{\partial T_{1}}{\partial x_{1}}+\sigma L_{s} \frac{\partial T_{1}}{\partial x_{3}}=0, \\
& \frac{\partial T_{1}}{\partial x_{2}}+\sigma L_{s} \frac{\partial T_{1}}{\partial x_{4}}=0, \\
& x_{2} \frac{\partial T_{1}}{\partial x_{1}}-x_{1} \frac{\partial T_{1}}{\partial x_{2}}+x_{4} \frac{\partial T_{1}}{\partial x_{3}}-x_{3} \frac{\partial T_{1}}{\partial x_{4}}=0 .
\end{aligned}
$$

The following equation is a consequence of the previous system:

$$
\left(x_{4}-\sigma L_{s} x_{2}\right) \frac{\partial T_{1}}{\partial x_{3}}-\left(x_{3}-\sigma L_{s} x_{1}\right) \frac{\partial T_{1}}{\partial x_{4}}=0 \text {. }
$$

in which at least one of the two $x$-dependent coefficients is nonzero. Note that, in the subregion of $V$ where both coefficients are nonzero, 
the system of PDE's implies that $T_{1}$ has to depend explicitly on all four components of the state. In any case, the above PDE's, together with the nonsingularity requirement for the matrix in (4.3), specify a set of transformation functions which yields exact state linearization. When these functions are used as outputs of the system, the conditions for full linearization with input-output decoupling are clearly satisfied. Even though it would be interesting to explore the nature of all the solutions to (4.2), this goes beyond the scope of the present paper. Here, it is interesting to note that neither of the following three functions of the state satisfies (4.2):

$T_{m}=x_{2} x_{3}-x_{1} x_{4}$

$$
I_{s}^{2}(x)=x_{1}^{2}+x_{2}^{2} \quad \Phi_{s}^{2}(x)=x_{3}^{2}+x_{4}^{2}
$$

Thus, the motor torque, the squared norm of the stator current and of the stator flux, when considered together, do not qualify as a set of outputs such that the full linearization problem can be solved. This can be expected also from physical reasons. Instead, a feasible and physically meaningful solution to (4.2) is found by choosing

$$
\begin{aligned}
& \frac{\partial T_{1}}{\partial x_{1}}=\sigma L_{s}\left(\sigma L_{s} x_{1}-x_{3}\right) \\
& \frac{\partial T_{1}}{\partial x_{2}}=\sigma L_{s}\left(\sigma L_{s} x_{2}-x_{4}\right),
\end{aligned}
$$

so that the following function is obtained:

$$
T_{1}(x)=\frac{1}{2}\left[\left(x_{3}-\sigma L_{s} x_{1}\right)^{2}+\left(x_{4}-\sigma L_{s} x_{2}\right)^{2}\right] \text {. }
$$

This function is proportional to the squared norm of the rotor flux, $\Phi_{r}{ }^{2}(x)$, and can be conveniently chosen as a system output. With this choice for $T_{1}(x)$, the other meaningful state functions mentioned in Section 2 can be used to complete the set of outputs (and, hence, of transforming coordinates $\left.\Psi_{j}(x)\right)$ :

$$
\mathrm{T}_{2}(\mathrm{x})=\mathrm{T}_{\mathrm{m}}(\mathrm{x}), \quad \mathrm{T}_{3}(\mathrm{x})=\mathrm{x}_{4} .
$$

Therefore, by defining the vector of system outputs as $y=h(x)=T(x)$,

full linearization of the system will be ensured.

\subsection{Sunthesis of the controller}

The proper application of nonlinear control techniques leads to a transparent two-stage solution: first, the control problem is converted into a set of linear independent single-input single-output ones; then, the controller synthesis is completed using any of the well-known control techniques on the linear equivalent system.

A decoupling and fully linearizing state-feedback can be derived by direct application of the decoupling algorithm. Using (4.6), from

$$
\begin{aligned}
& L_{g} h_{1}(x)=0, \\
& L_{g} L_{f} h_{1}(x)=\left[\begin{array}{lll}
\beta(1-\sigma)\left(x_{3}-\sigma L_{s} x_{1}\right) & \beta(1-\sigma)\left(x_{4}-\sigma L_{s} x_{2}\right) & 0
\end{array}\right] \neq 0, \\
& L_{g} h_{2}(x)=\left[\begin{array}{lll}
\left(x_{2}-x_{4} / \sigma L_{s}\right) & \left(x_{3} / \sigma L_{s}-x_{1}\right) & 0
\end{array}\right] \neq 0, \\
& L_{g} h_{3}(x)=\left[\begin{array}{lll}
0 & 1 & -x_{3}
\end{array}\right] \neq 0,
\end{aligned}
$$

it follows $p_{1}=\kappa_{1}-1=1, \rho_{2}=\kappa_{2}-1=0, p_{3}=\kappa_{3}-1=0$. The decoupling matrix $A(x)$ is then:

$$
A(x)=\left[\begin{array}{c}
L_{g} L_{f} h_{1}(x) \\
L_{g} h_{2}(x) \\
L_{g} h_{3}(x)
\end{array}\right] \text {, }
$$

that is the matrix (4.3) with $h(x)$ in place of $T(x)$. Its deteminant is $\operatorname{det} A(x)=x_{3} \beta(\sigma-1)\left[\left(x_{4}-\sigma L_{s} x_{2}\right)^{2}+\left(x_{3}-\sigma L_{s} x_{1}\right)^{2}\right] / \sigma L_{s}$.
Since $\beta \neq 0$ and $\sigma \neq 1$, matrix $A(x)$ is nonsingular (condition (D1)) if and only if $x_{3} \neq 0$ (nonzero d-component of the stiltor flux) and $h_{1}(x) \neq 0$ (nonzero rotor flux). These singularities define the region of validity of the proposed controller. A transfer from a given steadystate situation to another one should be mate in such at way to avoid the crossing of such singularities in the state-space. However, if the controller works properly, in the normal motor operation these singularities come never into pliay.

From the above derivation it follows immediately that $\sum_{i=1}^{3}\left(\rho_{i}+1\right)=4=n$,

which confirms that the decoupling state-feedback will also be a fully linearizing one (condition (D2)). This is obtained specifying $(\alpha(x), \beta(x))$ in

$$
u=\alpha(x)+\beta(x) v
$$

as

$$
\begin{aligned}
& \beta(x)=A^{-1}(x)=\left[\begin{array}{lll}
\frac{\left(x_{3}-\sigma L_{s} x_{1}\right)}{2 \beta(1-\sigma) h_{1}(x)} & -\frac{\sigma L_{s}\left(x_{4}-\sigma L_{s} x_{2}\right)}{2 h_{1}(x)} & 0 \\
\frac{\left(x_{4}-\sigma L_{s} x_{2}\right)}{2 \beta(1-\sigma) h_{1}(x)} & \frac{\sigma L_{s}\left(x_{3}-\sigma L_{s} x_{1}\right)}{2 h_{1}(x)} & 0 \\
\frac{\left(x_{4}-\sigma L_{s} x_{2}\right)}{2 x_{3} \beta(1-\sigma) h_{1}(x)} & \frac{\sigma L_{s}\left(x_{3}-\sigma L_{s} x_{1}\right)}{2 x_{3} h_{1}(x)} & -\frac{1}{x_{3}}
\end{array}\right], \\
& \alpha(x)=-A^{-1}(x)\left[\begin{array}{l}
L_{f}^{2} h_{1}(x) \\
L_{f} h_{2}(x) \\
L_{f} h_{3}(x)
\end{array}\right],
\end{aligned}
$$

with

$$
\begin{aligned}
L_{f}^{2} h_{1}(x)= & \beta \sigma\left\{\omega L_{s}(1-\sigma) h_{2}(x)-L_{s}[(\alpha+\beta)(1-\sigma)+4 \beta \sigma]\left(x_{1} x_{3}+x_{2} x_{4}\right)\right. \\
& \left.+L_{s}^{2}\left[\sigma(\alpha+2 \beta)-\alpha \sigma^{2}\right]\left(x_{1}^{2}+x_{2}^{2}\right)+\beta(1+\sigma)\left(x_{3}^{2}+x_{4}^{2}\right)\right\}, \\
L_{f} h_{2}(x)=-\left(\omega / \sigma L_{s}\right)\left(x_{3}^{2}+x_{4}^{2}\right)-(\alpha+\beta) h_{2}(x)+\omega\left(x_{1} x_{3}+x_{2} x_{4}\right), & \\
L_{f} h_{3}(x)= & -\left(\omega x_{3}+\alpha \sigma L_{s} x_{2}\right) .
\end{aligned}
$$

A closer look to the resulting controller shows the presence of physically interesting terms like the squared norm stator current, $\mathrm{I}_{s}{ }^{2}(x)$, and flux, $\Phi_{s}{ }^{2}(x)$, and the scalar product $I_{s}(x) \cdot \Phi_{s}(x)$; note that all of these quantities are independent from the orientation of the reference frame $(\mathrm{d}, \mathrm{g})$. The application of control law (4.8) to system (2.1) with outputs (4.6) yields a closed-loop system which, in the new coordinates $z$ specified by

$$
z_{1}=h_{1}(x), \quad z_{2}=L_{f} h_{1}(x), \quad z_{3}=h_{2}(x), \quad z_{4}=h_{3}(x),
$$

is in the linear controllable form (4.1) (see also Figure 1).

The control synthesis procedure is completed with the design of suitable inputs $v$ to be used in $(4.8)$ which, for convenience, may be rewritten as

$$
\begin{aligned}
& u_{1}=\beta_{11}(x) \gamma_{1}\left(v_{1}, \omega, x\right)+\beta_{12}(x) \gamma_{2}\left(v_{2}, \omega, x\right), \\
& u_{2}=\beta_{21}(x) \gamma_{1}\left(v_{1}, \omega, x\right)+\beta_{22}(x) \gamma_{2}\left(v_{2}, \omega, x\right), \\
& u_{3}=\beta_{31}(x) \gamma_{1}\left(v_{1}, \omega, x\right)+\beta_{32}(x) \gamma_{2}\left(v_{2}, \omega, x\right)+\left(-\frac{1}{x_{3}}\right)\left[v_{3}+\omega x_{3}+c_{7} x_{2}\right] .
\end{aligned}
$$

where 
$\gamma_{1}\left(v_{1}, \omega, x\right)=v_{1}+\omega c_{1} T_{m}(x)+c_{2} !_{s}(x) \cdot \Phi_{s}(x)+\left.c_{3}\right|_{s} ^{2}(x)+c_{4} \Phi_{s}^{2}(x)$

$\gamma_{2}\left(v_{2}, \omega, x\right)=v_{2}+\omega c_{5} \Phi_{s}^{2}(x)+c_{6} T_{m}(x)+\omega l_{s}(x) \cdot \Phi_{s}(x)$,

$\beta_{i j}(x)$ are elements of the $A^{-1}(x)$ matrix, and $c_{i}$ are constants including the machine parameters. Note that, for each measurement of the state $x$ and of $\omega$, a first estimate of the computations implied by (4.9) is of 29 products and 19 sums.

The appealing feature of the above control scheme is that the design of the $v_{i}, i=1,2,3$, can be performed independently for each controlled variable $y_{i}$, using only linear techniques such as pole placement, LQ optimal control or even root locus compensation and keeping in mind the given linear equivalent representation (4.1) of the closed-loop system.

As already illustrated, the control problem usually consists in:

- regulating the motor torque to a value $T_{m}=T_{m, c e s}$;

- keeping the squared norm of the rotor flux (normalized w.r.t. $\left.M^{2} / L_{r}{ }^{2}\right)$ at a desired value, $\Phi_{r, d e s}{ }^{2}$, which typically is assumed equal to the rated value;

- aligning the $d$-axis of the reference frame with the stator flux vector (i.e. constraining $\varphi_{\mathrm{qs}}=\mathrm{x}_{4}=0$ ).

The simplest choice is to design a PD controller for the rotor flux output and proportional ones for the motor torque and the $q$ component of the stator flux. As a result, the extemal inputs $v$, in terms of the original state variables $x$, are given by:

$$
\begin{aligned}
& v_{1}=\frac{K_{p}}{2}\left(\Phi_{r, \text { des }}^{2}-\Phi_{r}^{2}(x)\right)-K_{v}\left(\left.c_{8}\right|_{s}(x) \cdot \Phi_{s}(x)+\left.c_{9}\right|_{s} ^{2}(x)+c_{10} \Phi_{s}^{2}(x)\right), \\
& v_{2}=K_{m}\left(T_{m, \text { des }}-T_{m}(x)\right), \\
& v_{3}=-K_{q} x_{4},
\end{aligned}
$$

The gains $\left(K_{p}, K_{v}\right), K_{m}$ and $K_{q}$ may be independently adjusted to obtain the desired transient behaviour.

\section{Simulation results}

The proposed control approach has been tested on a high power induction motor, using as model parameters:

$\alpha=27.232 \mathrm{sec}, \quad \beta=17.697 \mathrm{sec}, \quad \sigma=0.064, L_{s}=0.179 \mathrm{H}$. The rated values of the stator and rotor fluxes are respectively equal to 7.3 and $6.88 \mathrm{~V} / \mathrm{sec}$ while the maximum torque is $1000 \mathrm{Nm}$.

A first set of simulation tests have been carried out supposing that the motor parameters are exactly known; the obtained results clearly confirm the decoupled and linear nature of the closed-loop system and, moreover, show that a sampling time of $1 \mathrm{~ms}$ does not induce significative errors.

In an actual drive the parameters of the machine are not exactly known; in particular, parameters $\alpha$ and $\beta$ are affected by large drifts during the normal motor operation. The range of variation for these quantities is usually in the order of $10 \%$ for $\alpha$ and $50 \%$ for $\beta$. In this situation, the input-output relation between $v$ and $y$ is no more exactly linear and decoupled.

Simulation runs using the maximum variations for the parameters have shown the robustness of the proposed approach. In fact, the simple control scheme (4.10) already gives acceptable results and the addition of an integral action leads to much better performance. Thus, a PID regulator has been used for the rotor flux and a PI one for the q-component of the stator flux. Simple proportional control was kept instead for the torque; generally, the reference signal for this variable is imposed by an outer loop closed around the load speed and so the need of a high-precision torque control is somewhat relaxed. The controller gains chosen for the tests are shown in Table $]$.

With these choices the performances of the complete controller are very satisfactory; the maximum errors on the relevant variables after a step change in the desired torque from 100 to $1000 \mathrm{Nm}$ are reported in Table 2 for a $10 \%$ higher value of $\alpha$ and a $50 \%$ higher value for $\beta$.
The peak values and the percentage deviations are all well within the acceptable ranges.

Figure 2 contains the time evolutions of the state, input and output variables for the case of a $50 \%$ variation of $\beta$ w.r.t. its nominal value. Note that the relevant variables which are not directly controlled (i.e. $I_{S}$ and $\Phi_{S}$ ) show a good behaviour.

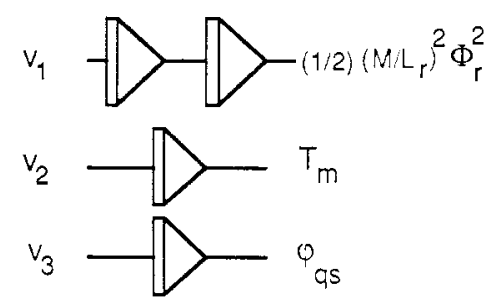

Figure 1 - Block diagran of input-output linear and decoupled relations

\begin{tabular}{|c|r|r|r|}
\hline & $K_{p}$ & $K_{i}$ & $K_{d}$ \\
\hline$\Phi_{r}$ & 235 & 450 & 22 \\
$\phi_{q s}$ & 180 & 900 & 0 \\
$T_{m}$ & 50 & 0 & 0 \\
\hline
\end{tabular}

Table 1 - PID gains used in the presence of parameter variations

\begin{tabular}{|l|c|c|c|c|}
\hline & $\Delta \Phi_{\mathrm{r}}$ & $\Delta \phi_{\mathrm{qS}}$ & $\Delta T_{\mathrm{m}}$ & $\Delta \Phi_{\mathrm{s}}$ \\
\hline$\Delta \alpha / \alpha=0.1$ & 0.05 & 0.02 & 58 & 0.26 \\
\hline$\Delta \beta / \beta=0.5$ & 0.15 & 0.003 & 200 & 0.27 \\
\hline
\end{tabular}

Table 2 - Maximum errors in the presence of parameter variations

\section{Conclusions}

It has been shown that the use of nonlinear control techniques based on the differential-geometric approach is effective for solving the control problem of induction motors. Exact linearization and inputoutput decoupling is possible by means of a static nonlinear statefeedback; among the advantages, the easy manipulation and tuning of control parameters that can be made on the linear side should be mentioned.

Simulation tests indicate a robust behaviour of the proposed controller in the presence of the typical parameter uncertainties of the machine; moreover, reasonable sampling times are allowed so that the use of digital signal processors is feasible.

A similar approach has been used very recently in [18], where a different definition of the motor inputs is assumed and no outputs are explicitly defined.

With the proposed approach it is possible to study in a systematic way different motor model and/or choices of input and output variables, in order to select the most convenient ones, e.g. for the purpose of an actual implementation. By using this methodology, some already known and widely used control techniques for induction motors, like field oriented control, can be recasted in the proper theoretical perspective. 

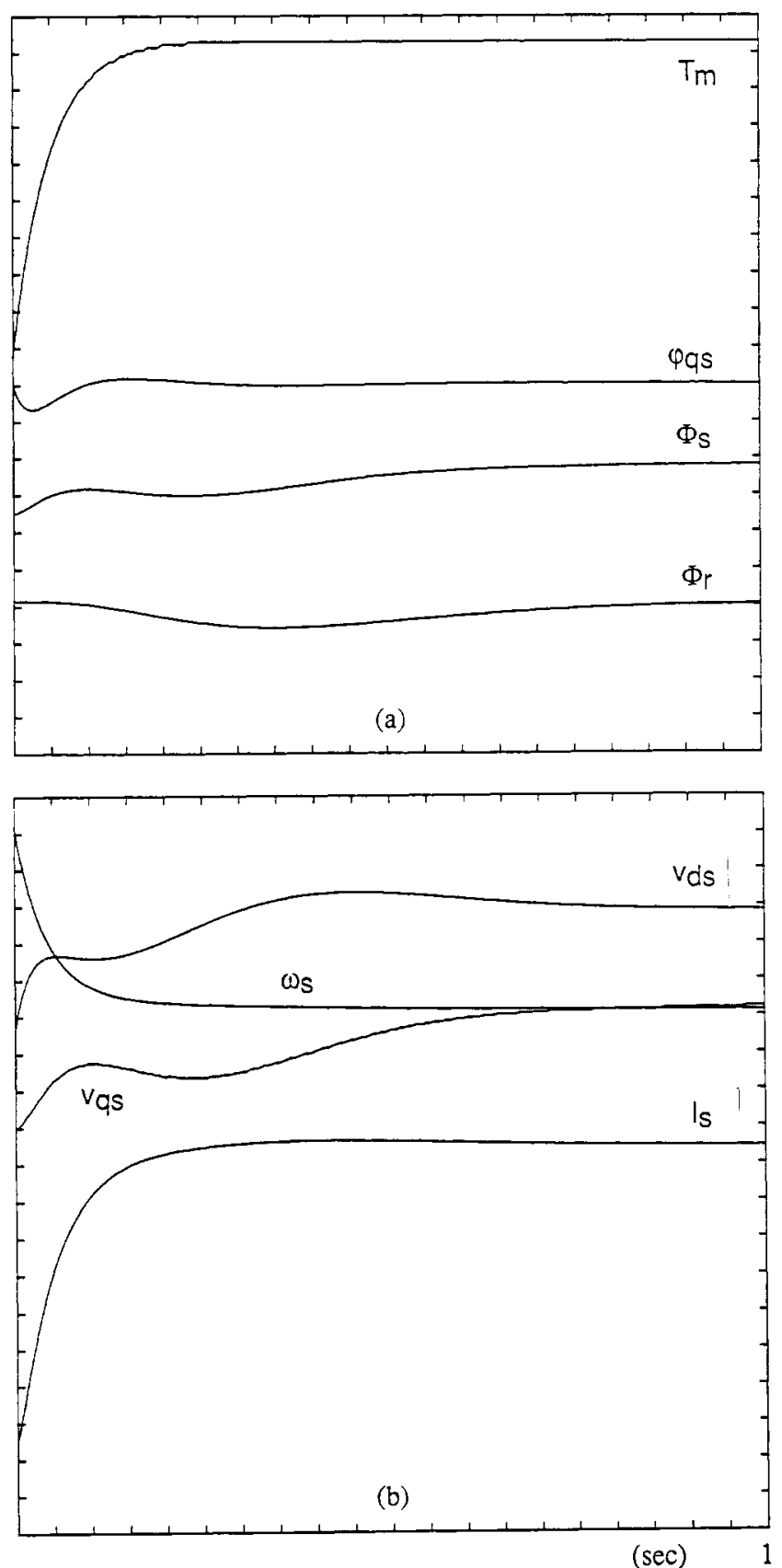

Figure 2 - Time evolution of relevant variables when $\Delta \beta / \beta=0.5$ (a) controlled outputs and stator flux (b) control inputs and stator current

Scales:

rotor flux $\Phi_{\mathrm{r}}$ motor torque $T_{m}$ q-comp. of stator flux $\varphi_{\mathrm{qs}}$ norm of stator flux $\Phi_{\mathrm{s}}$ d-comp. of stator voltage $v_{d s}$ $q$-comp. of stator voltage $v_{q s}$ slip angular speed $\omega_{\mathrm{s}}$ norm of stator current $I_{s}$

\section{References}

[1] Gabriel, R., Leonhard, W., Microprocessor Control of Induction Motor, Proc. Int. Semiconductor Power Converter Conf., Orlando (i982).

[2] Kuroe, Y., Haneda, H., Theory of Power-Electronic AC Motor Control for Modeling, Estimation, and Control and/or Analysis, 25th IEEE Conf. Decision and Control, Athens (1986).

[3] Leonhard, W., Control of Electrical Drives, Springer Verlag (1985).

[4] Blaschke, F., A New Mcthod for the Structural Decoupling of AC Induction Machine, Proc. 2nd IFAC Symp. on Multivariable Technical Control Systems, (1971).

[5] Bellini, A., Figalli, G., Ulivi, G., A Microcomputer Based Optimal Control System to Reduce the Effects of the Parameter Variations and Speed Measurements Errors in Induction Motor Drives, IEEE Trans. Ind. Appl., IA-22, 1 (1986).

[6] Figalli, G., La Cava, M., Tomasi, L., An Optimal Feedback Control for a Bilinear Model of Induction Motor Drives , Int. J. Control, 39, 5 (1984).

[7] Bellini, A., Figalli, G., La Cava, M. , A Field Oriented Adaptive Control of Induction Motors Useful to Reduce the Parameters' Variations and the Measurement Errors, Int. Conf, on Electrical Machines ICEM, (1984).

[8] Bellini, A., Figalli, G., Ulivi, G., A Microcomputer Based Direct Field Oriented Control of Induction Motors, Int. Conf. on Electrical Machines ICEM, Münich (1986).

[9] Hunt, L.R., Su, R., Meyer, G., Design for Multi-Input Nonlinear Systems, in: Differential Geometric Control Theory (R.W.Brockett, R.S.Millman, H.Sussmann Eds.), Birkhauser (1983).

[10] Isidori, A., Krener, A.J., Gori Giorgi, C., Monaco, S., Nonlinear Decoupling Via Feedback: A Differential Geometric Approach, IEEE Trans. Automatic Control, AC-26, 2 (1981).

[11] Jakubczyk, B., Respondek, W., On Linearization of Control Systems, Bull. Acad. Pol. Sci., Ser. Math. Astr. Phys., 28 (1980).

[12] Tarn, T.J., Bejczy, A.K., Isidori, A., Chen, Y., Nonlinear Feedback in Robot Arm Control, 23rd IEEE Conf. Decision and Control, Las Vegas (1984).

[13] Meyer, G., Su, R., Hunt, L.R., Application of Nonlinear Transformations to Automatic Flight Control, Automatica, 20, 1 (1984).

[14] Ilic'-Spong, M., Marino, R., Peresada, S.M., Taylor, D.G., Feedback Linearizing Control of Switched Reluctance Motors, IEEE Trans. Automatic Control, AC-32, 5 (1987).

[15] Bellini, A.,Figalli, G., Ulivi, G., A Microprocessor-Based State Observer for the Feedback Control of Induction Motor Drives Conf. Rec. EPE-85, (1985); to be published on Automatica.

[16] Verghese, G.C., Sanders, S.R., Observers for Faster Flux Estimation in Induction Machines, Proc. IEEE Power Electronics Specialist Conference, (1985).

[17] Isidori, A., Nonlinear Control Systems: An Introduction, Lecture Notes in Control and Information Sciences, 72, Springer Verlag (1985).

[18] Krzeminski, Z., Nonlinear Control of Induction Motors, 10th IFAC World Congress, Münich, July 1987. 